\section{Carsten Detka}

Otto von Guericke University Magdeburg, Germany

\section{Andrzej Piotrowski, Katarzyna Waniek}

Institute of Sociology

Faculty of Economics and Sociology

University of Lodz, Poland

\section{Professor Fritz Schütze - Work and Output}

DOI: https://doi.org/10.18778/1733-8077.10.1.09

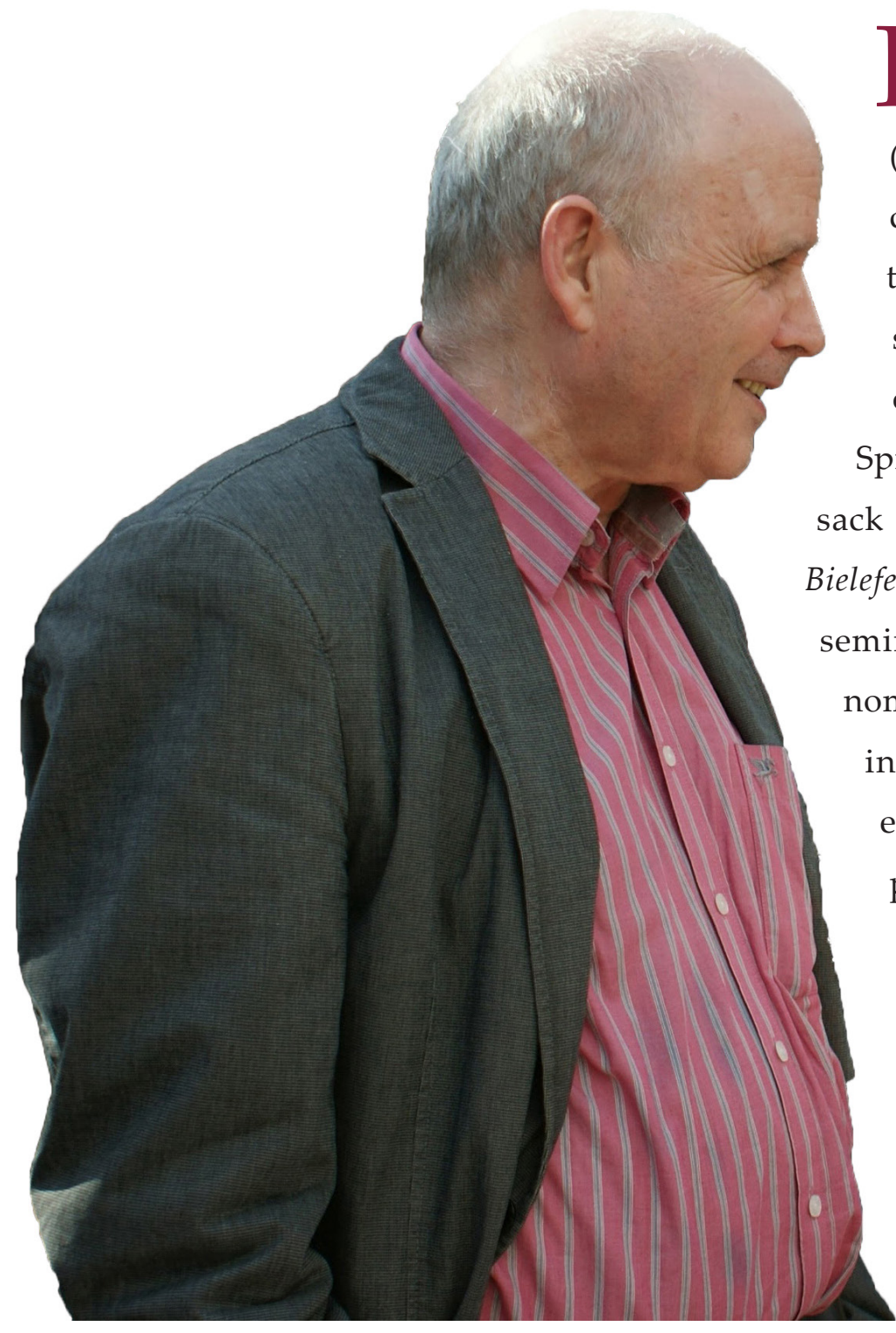

\section{Fritz Schütze}

(born in January 10, 1944) is a German sociologist. He is widely known for developing the autobiographical narrative interview research method. In the 70s, together with Joachim Matthes, Werner Meinefeld, Werner Springer, Ansgar Weymann, and Ralf Bohnsack (all belonging to so-called Arbeitsgruppe Bielefelder Soziologen), he has transplanted and disseminated symbolic interaction approach, ethnomethodology, and sociology of knowledge in Germany. Schütze is also very much interested in social work and so-called "modest" professions. He draws on the Chicago School tradition and promotes the grounded theory method elaborated by his mentor and friend - Anselm Strauss. Together with Gerhard Riemann he introduced the concept of trajectory to social sciences and has analysed the processes of suffering and self-alienation.
1964-1972: he studied sociology, philosophy, and general linguistics at the University of Münster.

1970-1972: he was a senior researcher at the University of Bielefeld.

In 1972 he received a $\mathrm{PhD}$ in the field of sociology from the University of Münster. His doctoral thesis was entitled: "Handeln in Sprache - Sprache im Handeln. Strategien des sprachbezogenen Denkens innerhalb und im Umkreis der Soziologie."

1972-1980: he was an academic assistant at the University of Bielefeld.

1978-1979: he was granted a habilitation scholarship of Deutsche Forschungsgemeinschaft (DFG) and pursued his research in the Department of Social and Behavioral Sciences at the University of California, San Francisco. Here, he cooperated with Anselm Strauss.

In $\mathbf{1 9 8 0}$ he received the venia legendi (lat. permission for lecturing) in the field of sociology from the Department of Sociology at the University of Bielefeld.

1980-1993: University Professor of qualitative methods in social research in the field of social work at the Gesamthochschule Kassel; since 1987 also teaching in the field of social studies.

1984-1985: he conducted his research in the Institute for Advanced Study in Princeton, NJ.

1993-2009: he was a Chair of General Sociology/Microsociology at the University of Magdeburg (now retired).
International Cooperation with Special Focus on Cooperation Between Professor Fritz Schütze and the University of Lodz

1986: $1^{\text {st }}$ visit of Professor Fritz Schütze (the Professor at the Gesamthochschule-Universität in Kassel) at the University of Lodz with lectures on the biographical method and his own approach to the method.

Since 1989 participation of members of the Department of Sociology of Culture at the University of Lodz (Zbigniew Bokszański, Marek Czyżewski, Andrzej Piotrowski, Alicja Rokuszewska-Pawełek, and Kaja Kaźmierska) in many international conferences and research workshops organized or co-organized by Professor Fritz Schütze.

1992-1993: Marek Czyżewski - Visiting Professor (for one academic year) at the Gesamthochschule-Universität in Kassel.

1993: Andrzej Piotrowski - Visiting Professor (for one academic term) at the Gesamthochschule-Universität in Kassel.

1995-1999: close cooperation (research projects, teaching and lecturing, scholarships for pursuing his habilitation) that resulted in the successful completion of the habilitation procedure. The topic of the habilitation thesis: "Öffentliche Kommunikation und Rechtsextremismus."

Since 1997 "Tri-National Research Platform: European Identity Work" - research workshops for researchers and students from Poland (University of 
Lodz), German (Otto-von-Guericke University in Magdeburg), and Wales (Bangor University), then also from Bamberg (Germany) and Belfast (Northern Ireland).

2003-2006: Leonardo da Vinci (no. 2003-D/03/B/F/ PP 146 087), “INVITE. New Ways of Biographical Counselling in Rehabilitative Vocational Training" (joined project run by four European universities in Finland, Germany, Poland, and Wales [Helsinki, Magdeburg, Lodz]).

2007: Katarzyna Waniek defended her doctoral thesis "Biographies and Identities of Young Polish Immigrants in Germany after 1989" written under the supervision of Professor Fritz Schütze.

2008-2011: Framework 7 Collaborative Project: “EUROIDENTITIES. The Evolution of European Identity: Using biographical methods to study the development of European Identity" (Grant Agreement no. 213998). Partners: Bulgarian Academy of Sciences in Sofia; Tallinn University of Technology, Estonia; University of Magdeburg, Germany; Federico II University, Naples, Italy; University of Lodz, Poland; Bangor University, Wales; Queen's University Belfast.

2012-2014: research project founded by the Polish-German Foundation for Science: “The People's Republic of Poland and the German Democratic Republic in memory and biographical experiences of people born between 1945-55. Sociological comparison based on biographical comparison." Partners: University of Lodz and Otto-von-Guericke University in Magdeburg.

\section{Research Projects in Germany}

2001-2002: “Prozessanalyse der Diabetes-Behandlung in Sachsen-Anhalt - eine qualitativ-sozialwissenschaftliche Untersuchung," AOK-Bundesvorstand.

2003-2004: "Zur Bedeutung der Akupunktur für AOK-Versicherte und ihre Ärztinnen und Ärzte im Rahmen des Bundesausschuss-Modellvorhabens," AOK-Bundesvorstand.

2009-2012: “Ärztliche Vermittlungs- und Beratungsmuster im Kontext lebensbedrohlicher Erkrankungen und ihre pädagogische Sensibilität. Die pädagogischen Komponenten der ärztlich-professionellen Informations-, Beratungs- und Betreuungsarbeit bei Herzinfarkt- und Brustkrebspatienten," DFG.

\section{Published Texts and Manuscripts}

1. 1973. (with Ralph Bohnsack) “Die Selektionsverfahren der Polizei in ihrer Beziehung zur Handlungskompetenz der Tatverdächtigen." Kriminologisches Journal 5(4):270-290.

2. 1973. (with Werner Meinefeld, Werner Springer, and Ansgar Weymann) "Grundlagentheoretische Voraussetzungen methodisch kontrollierten Fremdverstehens." Pp. 433-495 in Arbeitsgruppe Bielefelder Soziologen, Alltagswissen, Interaktion und gesellschaftliche Wirklichkeit, Vol. 2: Ethnotheorie und Ethnographie des Sprechens. Reinbek: Rowohlt.

3. 1975. Sprache soziologisch gesehen. Vol. 1, Strategien sprachbezogenen Denkens innerhalb und im Umkreis der Soziologie. Munich: Fink.
4. 1975. Sprache soziologisch gesehen. Vol. 2, Sprache als Indikator für egalitäre und nicht-egalitäre Sozialbeziehungen. Munich: Fink.

5. 1976. "Zur soziologischen und linguistischen Analyse von Erzählungen." Pp. 7-41 in Internationales Jahrbuch für Wissens - und Religionssoziologie, Vol. 10. Opladen: Westdeutscher Verlag.

6. 1976. (with Ralf Bohnsack, Werner Meinefeld, and Ansgar Weymann) "Theoretische und methodische Grundzüge kommunikativer Sozialforschung." Pp. 10-87 in Arbeitsgruppe Bielefelder Soziologen: Kommunikative Sozialforschung - Altagswissen und Alltagshandeln, Gemeindemachtforschung, Polizei, Politische Erwachsenenbildung. Munich: Fink.

7. 1976. "Zur Hervorlockung und Analyse von Erzählungen thematisch relevanter Geschichten im Rahmen soziologischer Feldforschung - dargestellt an einem Projekt zur Erforschung kommunaler Machtstrukturen." Pp. 159-260 in Arbeitsgruppe Bielefelder Soziologen: Kommunikative Sozialforschung - Alltagswissen und Alltagshandeln, Gemeindemachtforschung, Polizei, Politische Erwachsenenbildung. Munich: Fink.

8. 1977. (with Werner Kallmeyer) “Zur Konstitution von Kommunikationsschemata. Dargestellt am Beispiel von Erzählungen und Beschreibungen." Pp. 159-274 in Gesprächsanalysen, editet by D. Wegner. Hamburg: Buske.

9. 1977. “Die Technik des narrativen Interviews in Interaktionsfeldstudien: dargestellt an einem Projekt zur Erforschung von kommunalen Machtstrukturen." Arbeitsberichte und Forschungsmaterialien, Vol. 1. Bielefeld: Universität Bielefeld, Fakultät für Soziologie.
10. 1978. "Zur Konstitution sprachlicher Bedeutungen in Interaktionszusammenhängen." Pp. 98113 in Sprachstruktur - Sozialstruktur: zur linguistischen Theorienbildung, edited by U. Quasthoff. Königstein: Scriptor.

11. 1978. "Strategische Interaktion im Verwaltungsgericht - eine soziolinguistische Analyse zum Kommunikationsverlauf im Verfahren zur Anerkennung als Wehrdienstverweigerer." Pp. 67-156 in Schriften der Vereinigung für Rechtssoziologie, Vol 2: Interaktion vor Gericht, edited by W. Hassemer, W. Hoffmann-Riem, M. Weis. Baden-Baden: Nomos.

12. 1980. “Interaktionspostulate - Am Beispiel literarischer Texte." Pp. 72-94 in Literatur und Konversation. Sprachsoziologie und Pragmatik in der Literaturwissenschaft, edited by E. Hess-Lüttich. Wiesbaden: Akademische Verlagsgesellschaft Athenaion.

13. 1981. "Prozessstrukturen des Lebensablaufs." Pp. 67-156 in Biographie in handlungswissenschaftlicher Perspektive, edited by J. Matthes, A. Pfeifenberger, M. Stosberg. Nuremberg: Verlag der Nürnberger Forschungsvereinigung.

14. 1981. (with Joachim Matthes) "Zur Einführung: Alltagswissen, Interaktion und Gesellschaftliche Wirklichkeit." Pp. 11-53 in Alltagswissen, Interaktion und gesellschaftliche Wirklichkeit 1+2, edited by Arbeitsgruppe Bielefelder Soziologen. Opladen: Westdeutscher Verlag.

15. 1982. “Narrative Repräsentation kollektiver Schicksalsbetroffenheit." Pp. 568-590 in Erzählforschung Ein Symposion, eidited by E. Lämmert. Stuttgart: Metzler. 
16. 1983. "Biographieforschung und narratives Interview." Neue Praxis 13(3):283-293.

17. 1983. "Zur Praxisforschung im Supervisionsstudiengang. Drei Papiere." Pp. 23-60 in Beiträge zur Supervision. (Supervisionsforum WS 82/83), edited by N. Lippenmeier. Kassel: GesamthochschulBibliothek.

18. 1984. „Kognitive Figuren des autobiographischen Stegreiferzählens." Pp. 78-117 in Biographie und soziale Wirklichkeit. Neue Beiträge und Forschungsperspektiven, edited by M. Kohli et al. Stuttgart: Metzler.

19. 1984. "Professionelles Handeln, wissenschaftliche Forschung und Supervision." Pp. 262-389 in Beiträge zur Supervision 3 (Arbeitskonferenz "Theorie der Supervision," WS 83/84), edited by N. Lippenmeier. Kassel: Verlag der Universität-GHK.

20. 1987. "Situation." Pp. 157-164 in Sociolinguistics/ Soziolinguistik, edited by U. Ammon, N. Dittmar, K. J. Mattheier. Berlin, New York: Walter de Gruyter.

21. 1987. "Die Rolle der Sprache in der soziologischen Forschung." Pp. 413-431 in Sociolinguistics/ Soziolinguistik, edited by U. Ammon, N. Dittmar, K. J. Mattheier. Berlin, New York: Walter de Gruyter.

22. 1987. „Symbolischer Interaktionismus.” Pp. 520553 in Sociolinguistics/Soziolinguistik, edited by U. Ammon, N. Dittmar, K. J. Mattheier. Berlin, New York: Walter de Gruyter.

23. 1987. Das narrative Interview in Interaktionsfeldstudien, Kurseinheit 1. Hagen: Fernuniversität.
24. 1987. (with Gerhard Riemann) "Some Notes on a Students Workshop on 'Biography Analysis,' Interaction Analysis, and Analysis of Social Worlds." Biography and Society: Newsletter of the International Sociological Association Research Committee 38(8):54-70.

25. 1987. (conference paper) "Interaktionsanalyse von Supervisionsprozessen: Supervision als erkenntnisgenerierendes Verfahren." Pp. 377-380 in 23. Deutscher Soziologentag 1986 in Hamburg: Beiträge der Sektions- und Ad-hoc-Gruppen, edited by J. Friedrichs. Opladen: Westdt.

26. 1988. "Professional Schools: Ein Entwicklungspotential für die Zukunft der GHK." Gießhausgespräche (8). Kassel: Verlag der UniversitätGHK.

27. 1989. “Kollektive Verlaufskurve und kollektiver Wandlungsprozeß. Dimensionen des Vergleichs von Kriegserfahrungen amerikanischer und deutscher Soldaten im Zweiten Weltkrieg." BIOS 1/89:31-109.

28. 1990. “Presja i wina: doświadczenia młodego żołnierza niemieckiego w czasie drugiej wojny światowej i ich implikacje biograficzne." Pp. 325-339 in Metoda biograficzna w socjologii, edited by J. Włodarek, M. Ziółkowski. Translated into Polish by M. Ziółkowska. Warszawa: PWN.

29. 1991. (unpublished manuscript) "Autobiographical accounts of war experiences: An outline for the analysis of topically focused autobiographical texts - using the example of the ‘Robert Rasmus' account in Studs Terkel's book, 'The Good War."' University of Kassel.
30. 1991. (with Gerhard Riemann) “'Trajectory' as a Basic Concept for Analyzing Suffering and Disorderly Social Processes." Pp. 333-357 in Social Organization and Social Structure. Essays in Honour of Anselm Strauss, edited by D. Maines. New York: Aldine de Gruyter.

- Translated into Polish by Z. Bokszański and A. Piotrowski: “'Trajektoria' jako podstawowa koncepcja teoretyczna $\mathrm{w}$ analizach cierpienia i bezładnych procesów społecznych."

- 1992. Kultura i Społeczeństwo 39(2):89-109.

- 2012. Pp. 389-414 in Metoda biograficzna $\mathrm{w}$ socjologii. Antologia tekstów, edited by K. Kaźmierska. Cracow: Nomos.

31. 1991. “Biographieanalyse eines Müllerlebens - Innovationsbereitschaft als Familientradition und Lebensführungshabitus: Wie die Müllerfamilie Berger die Krisen des Mühlensterbens um die Jahrhundertwende und in den Fünfziger Jahren überwunden hat." Pp. 206-227 in Wasser- und Windmühlen in Kurhessen und Waldeck- Pyrmont, edited by H. Scholz. Kaufungen: Axel Eibel.

32. 1992. "Pressure and Guilt: War Experiences of a Young German Soldier and their Biographical Implications (Part I)." International Sociology 7(2):187-208.

33. 1992. "Pressure and Guilt: War Experiences of a Young German Soldier and Their Biographical Implications (Part II)." International Sociology 7(3):347-367.

34. 1992. "Sozialarbeit als 'bescheidene' Profession." Pp. 132-170 in Erziehen als Profession. Zur Logik professionellen Handelns in pädagogischen Feldern, edited by B. Dewe, W. Ferchhoff, F. Radtke. Opladen: Leske and Budrich.

35. 1993. (with Claudia Lützen and Ulrike Schulmeyer-Herbold) “Unterschiede in der Berichterstattung der FR und der FAZ zu studentischen Anliegen 1967/68 und 1989/90 - Eine qualitative Auswertung." Pp. 300-341 in Phantasie und Realität in der Spätadoleszenz. Gesellschaftliche Veränderungen und Entwicklungsprozesse bei Studierenden, edited by M. Leuzinger-Bohleber, E. Mahler. Opladen: Westdeutscher Verlag.

36. 1993. “Die Fallanalyse. Zur wissenschaftlichen Funclierung einer klassischen Methode der Sozialen Arbeit." Pp. 191-221 in Der sozialpädagogische Blick. Lebensweltorientierte Methoden in der sozialen Arbeit, edited by T. Rauschenbach, F. Ortmann, M. Karsten. Weinheim, Munich: Juventa.

37. 1994. "Strukturen des professionellen Handelns, biographische Betroffenheit und Supervision." Supervision 26:10-39.

38. 1994. "Das Paradoxe in 'Felix' Leben als Ausdruck eines 'wilden' Wandlungsprozesses." Pp. 13-60 in Biographie als Text, edited by H. Koller, R. Kokemohr. Weinheim: Deutscher Studien Verlag.

39. 1994. "Ethnographie und sozialwissenschaftliche Methoden der Feldforschung. Eine mögliche methodische Orientierung in der Ausbildung und Praxis der Sozialen Arbeit?" Pp. 189-297 in Modernisierung Sozialer Arbeit durch Methodenentwicklung und - reflexion, edited by N. Groddeck, M. Schumann. Freiburg: Lambertus. 
40. 1995. "Verlaufskurven des Erleidens als Forschungsgegenstand der interpretativen Soziologie." Pp. 116-157 in Erziehungswissenschaftliche Biographieforschung, edited by H. Krüger, W. Marotzki. Wiesbaden: Leske and Budrich.

- Translated into Polish by M. Czyżewski: “Trajektorie cierpienia jako przedmiot badań socjologii interpretatywnej."

- 1997. Studia Socjologiczne 114(1):11-57.

- 2012. Pp. 415-458 in Metoda biograficzna $w$ socjologii. Antologia tekstów, edited by K. Kaźmierska. Cracow: Nomos.

41. 1996. “Organisationszwänge und hoheitsstaatliche Rahmenbedingungen im Sozialwesen: Ihre Auswirkung auf die Paradoxien des professionellen Handelns." Pp. 183-275 in Pädagogische Professionalität. Untersuchungen zum Typus pädagogischen Handelns, edited by A. Combe, W. Helsper. Frankfurt: Suhrkamp.

42. 1997. “Kognitive Anforderungen an das Adressatendilemma in der professionellen Fallanalyse der Sozialarbeit (Cognitive Requirements Regarding the Dilemma whom to Address in Professional Case Analysis of Social Work)." Pp. 39-60 in Rekonstruktive Sozialpädagogik. Konzepte und Methoden sozialpädagogischen Verstehens in Forschung und Praxis (Re-Constructive Social Pedagogy. Concepts and Methods of Socio-Pedagogical Understanding in Research and Practice), edited by G. Jakob, H. Wensierski. Weinheim and Munich: Juventa.

43. 2000. "Schwierigkeiten bei der Arbeit und Paradoxien des professionellen Handelns. Ein grundlagentheoretischer Aufriß." Zeitschrift für qualitative Bildungs-, Beratungs- und Sozialforschung 1(1):49-96.

44. 2001. “Ein biographieanalytischer Beitrag zum Verständnis von kreativen Veränderungsprozessen: die Kategorie der Wandlung." Pp. 137162 in Materialität des Geistes. Zur Sache Kultur - Im Diskurs mit Ulrich Oevermann, edited by R. Burkholz, C. Gärtner, F. Zehentreiter. Weilerswist: Velbrück-Verlag.

45. 2001. "Rätselhafte Stellen im narrativen Interview." Handlung Kultur Interpretation 10(1):12-28.

46. 2001. (unpublished manuscript) "Zur trinationalen Zusammenarbeit zwischen den Universitäten Lodz, Wales/Bangor und Magdeburg im Bereich der Mikrosoziologie und Kulturstudien, Magdeburg."

47. 2002. “Das Konzept der sozialen Welt im symbolischen Interaktionismus und die Wissensorganisation in modernen Komplexgesellschaften." Pp. 57-84 in Soziale Welten und kommunikative Stile: Festschrift für Werner Kallmeyer zum 60. Geburtstag, Studien zur Deutschen Sprache, no. 22, edited by I. Keim, W. Schütte, W. Kallmeyer. Teubingen: Narr.

- Translated into Polish by A. Pawlak and K. Waniek: “Koncepcja świata społecznego $\mathrm{w}$ symbolicznym interakcjonizmie oraz organizacja wiedzy w nowoczesnych złożonych społeczeństwach." Pp. 489-514 in Metoda biograficzna w socjologii. Antologia tekstów, edited by K. Kaźmierska. Cracow: Nomos.

48. 2002. (with Carsten Detka, Monika Müller) Zwischenbericht zum Forschungsprojekt Prozessanalyse der Diabetes-Behandlung in Sachsen-Anhalt. Eine qualitativ-sozialwissenschaftliche Untersuchung." Magdeburg: ISOZ. Otto-von-Guericke-Universität Magdeburg.

49. 2004. "Hülya's Migration to Germany as SelfSacrifice Undergone and Suffered in Love for Her Parents, and Her Later Biographical Individualization. Biographical Problems and Biographical Work of Marginalisation and Individualisation of a Young Turkish Woman in Germany (Part I)." Forum Qualitative Social Research 4(3). (http://www.qualitative-research.net/index.php/ fqs/article/view/671).

50. 2004. (with Lorenza Mondada) "Soziale Interaktion und die Herstellung von Wissenschaft: Einführung in den Themenschwerpunkt." Zeitschrift für qualitative Bildungs-, Beratungs- und Sozialforschung 5(2):139-154.

51. 2005. (with Michaela Frohberg, Jürgen Voigt, Carsten Detka, Birthe Dorendorf, and Christian Malina) Kurzgefasste Zusammenfassung der Ergebnisse des Magdeburger Akupunkturprojektes. Magdeburg: ISOZ. Otto-von-Guericke-Universität Magdeburg.

52. 2005. "Eine sehr persönlich generalisierte Sicht auf qualitative Forschung." Zeitschrift für qualitative Bildungs-, Beratungs- und Sozialforschung 6(2):211-248.

53. 2006. (with Werner Fiedler, Jörg Frommer, Werner Helsper, Heinz-Hermann Krüger, Winfried Marotzki, and Ursula Rabe-Kleberg) Studien zur qualitativen Bildungs-, Beratungs- und Sozialforschung. Opladen: Budrich.
54. 2006. (with Katrin Perleberg and Viktoria Heine) "Sozialwissenschaftliche Biographieanalyse von chronisch kranken Patientinnen auf der empirischen Grundlage des autobiographischnarrativen Interviews - exemplifiziert an der Lebensgeschichte einer jungen Patientin mit Morbus Crohn." Psychotherapie \& Sozialwissenschaft 8(1):95-145.

55. 2007. (with Detlef Garz and Sandra Tiefel Sandra) "An alle, die Deutschland vor und während Hitler gut kennen: autobiographische Beiträge deutscher Emigranten zum wissenschaftlichen Preisausschreiben der Harvard University aus dem Jahr 1939." Zeitschrift für Qualitative Forschung 8(2):179-188.

56. 2007. (with Sandra Betts, Aled Griffiths, and Peter Straus) "Biographical Counselling: an Introduction." INVITE - Biographical Counselling in Rehabilitative Vocational Training - Further Education Curriculum. (http://www.uni-magdeburg.de/ zsm/projekt/biographical/1/0.pdf).

57. 2008. “Biography Analysis on the Empirical Base of Autobiographical Narratives: How to Analyse Autobiographical Narrative Interviews." European Studies on Inequalities and Social Cohesion 1/2 (part I) and 3/4 (part II).

- Translated into Polish by Katarzyna Waniek: "Analiza biograficzna ugruntowana empirycznie $\mathrm{w}$ autobiograficznym wywiadzie narracyjnym. Jak analizować autobiograficzne wywiady narracyjne. Część 1." Pp. 141-276 in Metoda biograficzna w socjologii. Antologia tekstów, edited by K. Kaźmierska. Cracow: Nomos. 
58. 2008. "Ansprache beim Akademischen Gottesdienst am 19. Januar 2001 in der Wallonerkirche zu Magdeburg." Pp. 73-99 in Predigten der Magdeburger Akademischen Gottesdienste 2001 bis 2007, edited by G. Gademann, H. Schultze, H. Kaffka. Magdeburg: Evangelischen Hochschulbeirats Magdeburg.

59. 2009. “Die Berücksichtigung der elementaren Dimensionen biografischer Arbeit in der Schule der Zukunft." Pp. 359-364 in Schule 2020 aus Expertensicht, edited by D. Bosse, P. Posch. Wiesbaden: VS Verlag für Sozialwissenschaften.

60. 2009. "Einführung: Subjektivität im Kontext der Forschung zum Expertentum." Pp. 91-101 in Subjektivität in der qualitativen Forschung, edited by V. Kubek. Opladen: VS Verlag für Sozialwissenschaften.

61. 2009. "European identity work - first draft of a proposal for a joint research project." Przegląd Socjologiczny 57(2):9-44.

62. 2009. “Nachruf: Joachim Matthes (1. Juni 1930 - 3. Mai 2009)." Zeitschrift für Soziologie 38(5):441-444.

63. 2009. (with K. Schlöpker, Markus Herrmann, Carina Großer-Kaya, Bernt-Peter Robra, Barbara Dippelhofer-Stiem, and Christoph Heintze) “Problemlagen von versteckt lebenden Migranten in Deutschland: Analyse der medizinischen Beratungsanlässe in Berlin, Bonn und Köln." Das Gesundheitswesen 71(12):839-844.

64. 2010. (with Lena Inowlocki and Gerhard Riemann) "Das forschende Lernen in der Biographieforschung - europäische Erfahrungen." Zeitschrift für qualitative Forschung 11(2):183-195.
65. 2011. "Europäische Orientierungs- und Identitätsarbeit aus der Sicht europa-sensibilisierter Bürger der Europäischen Union ü Aufriss eines Forschungsprojektes." Pp. 475-504 in Biographie und Gesellschaft, edited by H. Herzberg, E. Kammler. Frankfurt: Campus-Verl.

66. 2011. (with Robert Miller) "The Evolution if European Identity: Using Biographical Methods to study the Development of European Identity." Przeglad Socjologiczny 60(1):9-40.

67. 2011. (with Anja Schröder-Wildhagen) "How to Deal with Autobiographical Narrative Interviews in the Euroidentity Research Project." Przeglad Socjologiczny 60(1):41-91.

68. 2012. (with Anja Schröder-Wildhagen, Ulrike Nagel, and Bärbel Treichel) "Discoverers in European Mental Space: The Biographical Experiences of Participants in European Civils Society Organizations." Pp. 150-169 in The Evolution of European Identities. Biographical Approaches, edited by R. Miller, G. Day. Houndmills: Palgrave MacMillan.

69. 2012. (with Anja Schröder-Wildhagen, Ulrike Nagel, and Bärbel Treichel): “Miriam, an Environmental Activist." Pp. 170-181 in The Evolution of European Identities. Biographical Approaches, edited by R. Miller, G. Day. Houndmills: Palgrave MacMillan.

70. 2012. (with Anja Schröder-Wildhagen) “European Mental Space and its Biographical Relevance." Pp. 255-278 in The Evolution of European Identities. Biographical Approaches, edited by R. Miller, G. Day. Houndmills: Palgrave MacMillan.
71. 2012. (with Gerhard Riemann) “Die soziologische Komplexität der Fallanalyse von Mary Richmond." Pp. 131-201 in Forschungstraditionen der Sozialen Arbeit, edited by K. Bromberg, W. Hoff, I. Miethe. Opladen: Budrich.

72. 2012. (with Viktoria Heine, Michael Koehler, and Jörg Frommer) "From life-threatening experiences to ideas of rescue: coping with 'trajectories of suffering' in adult acute leukaemia survivors." Research in Psychotherapy 15(2):107-116.

73. 2012. (with Lena Inowlocki, Ulrike Nagel, Gerhard Riemann, Anja Schröder-Wildhagen, and Bärbel Treichel) “Policy Suggestions Regarding Support of the Work of European Civil Society Organisations." Przeglad Socjologiczny 61(4):9-76.

74. 2012. "Biographical Process Structures and Biographical Work in a Life of Cultural Marginality and Hybridity: Don Decker's Autobiographi- cal Account." Pp. 159-242 in Don Decker's Apache Odyssey, edited by B. Treichel, G. Bartelt. Berlin: Frank \& Timme.

75. 2013. “Alltägliche Kategorisierungs-, Typisierungs- und Klassifikationstätigkeit der Ärzte als abgekürzte professionelle Erkenntnis- und Vermittlungszuwendung." Pp. 227-290 in Der soziale Körper, edited by H. Herzberg, A. Seltrecht. Opladen: Budrich.

76. 2013. (with Kaja Kaźmierska) “Wykorzystanie autobiograficznego wywiadu narracyjnego w badaniach nad konstruowaniem obrazu przeszłości w biografii. Na przykładzie socjologicznego porównania narracji na temat życia w PRL i NRD." Przeglad Socjologii Jakościowej 9(4):122-139.

77. (no publishing date, unpublished manuscript) "Outline for the Method of Biography Analysis." 